\title{
Multiscale methods with compactly supported radial basis functions for elliptic partial differential equations on bounded domains
}
A. Chernih ${ }^{1}$
Q. T. Le Gia ${ }^{2}$

(Received 29 October 2012; revised 22 March 2013)

\begin{abstract}
We propose a multiscale approximation method for constructing numerical solutions to elliptic partial differential equations on a bounded domain. The approximate solution is constructed in a multi-level fashion, with each level using compactly supported radial basis functions on an increasingly fine mesh. Numerical experiments support the theoretical results.

Subject class: $65 \mathrm{~N} 35,65 \mathrm{~N} 15$

Keywords: multiscale, collocation, radial basis function, elliptic partial differential equation

http://journal . austms.org.au/ojs/index.php/ANZIAMJ/article/view/6304 gives this article, (c) Austral. Mathematical Soc. 2013. Published May 13, 2013, as part of the Proceedings of the 16th Biennial Computational Techniques and Applications Conference. ISSN 1446-8735. (Print two pages per sheet of paper.) Copies of this article must not be made otherwise available on the internet; instead link directly to this URL for
\end{abstract} this article. 


\section{Contents}

1 Introduction

C138

2 Preliminaries

C139

3 Multiscale collocation

C141

4 Numerical experiments

C148

5 Conclusion

C150

References

C151

\section{Introduction}

Radial basis functions (RBF) are increasingly important in the area of approximation theory. For solving partial differential equations (PDE) using RBF, meshless collocation methods [6, 4] are more often used than Galerkin methods $[10,11]$. Two excellent recent books covering practical and theoretical issues are by Fasshauer [4] and Wendland [12]. A function $\Phi: \mathbb{R}^{\mathrm{d}} \rightarrow \mathbb{R}$ is said to be radial if there exists a function $\phi:[0, \infty) \rightarrow \mathbb{R}$ such that $\Phi(\mathbf{x})=\phi\left(\|\mathbf{x}\|_{2}\right)$ for all $\mathbf{x} \in \mathbb{R}^{\mathrm{d}}$, where $\|\cdot\|_{2}$ denotes the usual Euclidean norm in $\mathbb{R}^{\mathrm{d}}$. In this case, we define an RBF for a given centre $\mathbf{x}_{i} \in \mathbb{R}^{\mathrm{d}}$ as

$$
\Phi_{\mathfrak{i}}(\mathbf{x})=\phi\left(\left\|\mathbf{x}-\mathbf{x}_{\mathbf{i}}\right\|_{2}\right) .
$$

A practical issue concerns the scale factor to use for the RBF [8, 5]. A small scale leads to a sparse and consequently well conditioned linear system, but at the price of the approximation power. Conversely, a large scale has better approximation power, but at the price of a poorly conditioned linear system. The multiscale algorithms proposed in this article are constructed over multiple levels. The residual of the current stage is the target function for the next 
stage. Later stages use, as basis functions, RBF with smaller support and more closely spaced centres.

Le Gia et al. [7] gave an analysis of multiscale algorithms for RBF collocation for a sphere. The extension to a bounded domain changes the analysis significantly as boundary effects now need to be considered. The sufficient conditions for convergence, presented here, are the main contribution of this article and are significant. A similar algorithm was studied by Fasshauer [4], although numerical experiments only showed limited convergence. The theory presented here shows that this limited convergence was due to the parameter choices. In contrast, our numerical experiments converge at every level. Numerical experiments with multiscale algorithms with compactly supported RBF for elliptic PDE were also presented by Chen et al. [2], although without any theoretical results.

In the next section we provide the concepts involved in our multiscale algorithm and provide necessary background material regarding point sets and function theory. Section 3 deals with the symmetric collocation algorithm and Section 4 provides numerical experiments to test the theoretical results.

\section{Preliminaries}

We use (scaled) compactly supported RBF to construct multiscale approximate solutions to PDE, that is, we form the solution over multiple levels. We work with a given domain $\Omega \subseteq \mathbb{R}^{\mathrm{d}}$.

At each level we have a finite point set $X \subseteq \Omega$. We define the mesh norm as

$$
\mathbf{h}_{\mathbf{X}, \Omega}:=\sup _{\mathbf{x} \in \Omega} \min _{\mathbf{x}_{j} \in X}\left\|\mathbf{x}-\mathbf{x}_{\mathbf{j}}\right\|_{2},
$$

which is a measure of the uniformity of the points in $X$ with respect to $\Omega$. At each level $i$ we denote the mesh norm by $h_{i}$. 
For a given domain $\Omega \subseteq \mathbb{R}^{\mathrm{d}}$, with a given $k \in \mathbb{N}_{0}$ and $1 \leqslant p<\infty$, the Sobolev space $W_{p}^{k}(\Omega)$ consist of all $u$ with weak derivatives $D^{\alpha} u \in L_{p}(\Omega),|\alpha| \leqslant k$. The semi-norms and norms are defined as

$$
|u|_{W_{\mathfrak{p}}^{k}(\Omega)}=\left(\sum_{|\alpha|=k}\left\|D^{\alpha} u\right\|_{L_{p}(\Omega)}^{p}\right)^{1 / p}, \quad\|u\|_{W_{\mathfrak{p}}^{k}(\Omega)}=\left(\sum_{|\alpha| \leqslant k}\left\|D^{\alpha} u\right\|_{L_{p}(\Omega)}^{p}\right)^{1 / p} .
$$

For $p=\infty$ these definitions become

$$
|u|_{W_{\infty}^{k}(\Omega)}=\sup _{|\alpha|=k}\left\|D^{\alpha} u\right\|_{L_{\infty}(\Omega)}, \quad\|u\|_{W_{\infty}^{k}(\Omega)}=\sup _{|\alpha| \leqslant k}\left\|D^{\alpha} u\right\|_{L_{\infty}(\Omega)} .
$$

For the case $p=2$ we write $W_{2}^{k}(\Omega)=H^{k}(\Omega)$.

The functions that we are concerned with are defined on a bounded domain $\Omega$ with a Lipschitz boundary. As a result, there is an extension operator for functions defined in Sobolev spaces which is presented in the following lemma [1, Theorem 1.4.5]. Further details were provided by Stein [9].

Lemma 1. Suppose $\Omega \subseteq \mathbb{R}^{\mathrm{d}}$ has a Lipschitz boundary. Then there is an extension mapping $\mathrm{E}: \mathrm{H}^{\tau}(\Omega) \rightarrow \mathrm{H}^{\tau}\left(\mathbb{R}^{\mathrm{d}}\right)$ defined for all non-negative integers $\tau$ satisfying $\left.\mathrm{E} v\right|_{\Omega}=v$ for all $v \in \mathrm{H}^{\tau}(\Omega)$ and

$$
\|E v\|_{H^{\tau}\left(\mathbb{R}^{\mathrm{d}}\right)} \leqslant \mathrm{C}\|v\|_{H^{\tau}(\Omega)} .
$$

Here, $\mathbf{C}$ denotes a generic constant.

We use the Wendland compactly supported RBF [12] with a (level-specific) scaling parameter $\delta>0$. We define the scaled kernels as

$$
\Phi_{\delta}(\mathbf{x}):=\delta^{-\mathrm{d}} \Phi\left(\frac{\mathbf{x}}{\delta}\right) \text {. }
$$

For the Wendland's functions there exist two constants $0<\mathrm{c}_{1} \leqslant \mathrm{c}_{2}$ such that the Fourier transform of $\Phi, \widehat{\Phi}$, satisfies [12]

$$
c_{1}\left(1+\|\boldsymbol{\omega}\|_{2}^{2}\right)^{-\tau} \leqslant \widehat{\Phi}(\boldsymbol{\omega}) \leqslant c_{2}\left(1+\|\boldsymbol{\omega}\|_{2}^{2}\right)^{-\tau}, \quad \boldsymbol{\omega} \in \mathbb{R}^{\mathrm{d}} .
$$


The native space of the Wendland's functions $\mathcal{N}_{\Phi}\left(\mathbb{R}^{\mathrm{d}}\right)$ is norm equivalent to the Sobolev space $\mathrm{H}^{\tau}\left(\mathbb{R}^{\mathrm{d}}\right)$. Consequently, the Fourier transform of $\Phi_{\delta}$, $\widehat{\Phi_{\delta}}(\boldsymbol{\omega})=\widehat{\Phi}(\delta \boldsymbol{\omega})$ satisfies

$$
c_{1}\left(1+\delta^{2}\|\boldsymbol{\omega}\|_{2}^{2}\right)^{-\tau} \leqslant \widehat{\Phi_{\delta}}(\boldsymbol{\omega}) \leqslant c_{2}\left(1+\delta^{2}\|\boldsymbol{\omega}\|_{2}^{2}\right)^{-\tau}, \quad \boldsymbol{\omega} \in \mathbb{R}^{\mathrm{d}} .
$$

We require norm equivalence as stated in the following lemma of Chernih and Le Gia [3].

Lemma 2. For every $\delta \in\left(0, \delta_{\mathrm{a}}\right]$ and for all $\mathrm{g} \in \mathrm{H}^{\tau}\left(\mathbb{R}^{\mathrm{d}}\right)$ there exist constants $0<\mathrm{c}_{3} \leqslant \mathrm{c}_{4}$ such that

$$
c_{3}\|g\|_{\Phi_{\delta}} \leqslant\|g\|_{H^{\tau}\left(\mathbb{R}^{d}\right)} \leqslant c_{4} \delta^{-\tau}\|g\|_{\Phi_{\delta}} .
$$

\section{Multiscale collocation}

In this section we consider the PDE

$$
\mathcal{L} u(x)=f(x), \quad x \text { in } \Omega,
$$

with

$$
\mathcal{B}(\mathbf{x})=\mathrm{g}(\mathbf{x}), \quad \mathbf{x} \text { on } \partial \Omega,
$$

where $\Omega \subseteq \mathbb{R}^{\mathrm{d}}$ is a bounded domain with a $C^{\mathrm{k}, \mathrm{s}}$ boundary $\partial \Omega$, with $\mathrm{k} \in \mathbb{N}_{0}$ and $s \in[0,1)$. The differential operator $\mathcal{L}$ is elliptic and self-adjoint of order $m$, for some $\mathrm{m}>0$, for which we assume there exist constants $0<c_{5} \leqslant c_{6}$ such that

$$
c_{5}\left(1+\|\boldsymbol{\omega}\|_{2}^{2}\right)^{m / 2} \leqslant \widehat{\mathcal{L}}(\boldsymbol{\omega}) \leqslant c_{6}\left(1+\|\boldsymbol{\omega}\|_{2}^{2}\right)^{m / 2} .
$$

The operator $\mathcal{B}$ is a boundary operator.

Suppose that $\Phi$ is a kernel that satisfies condition (2) for some $\tau>m+d / 2$. This assumption ensures that we may apply $\mathcal{L}$ twice to one of the arguments of $\Phi$ and still have a continuous function. We choose interior and 
boundary point sets as $X=X_{1} \cup X_{2}$ where $X_{1}=\left\{x_{1}, \ldots, x_{n}\right\} \subseteq \Omega$ and $\mathbf{X}_{2}=\left\{\mathbf{x}_{n+1}, \ldots, \mathbf{x}_{\mathrm{N}}\right\} \subseteq \partial \Omega$ and we construct our approximation

$$
\widetilde{u}=\sum_{j=1}^{n} \alpha_{j} \mathcal{L}_{2} \Phi\left(\left\|\cdot-x_{j}\right\|_{2}\right)+\sum_{j=n+1}^{N} \alpha_{j} \mathcal{B}_{2} \Phi\left(\left\|\cdot-x_{j}\right\|_{2}\right),
$$

where the subscript of 2 on $\mathcal{L}$ and $\mathcal{B}$ indicates that these operators act with respect to their second argument. The mesh norms of $X_{1}$ and $X_{2}$ are given by $h_{1}$ and $h_{2}$, respectively.

Without loss of generality, we only consider the case where the RBF centres coincide with the collocation points. In this case, solving (4) and (5) by collocation on the set $X_{1}$ is equivalent to selecting $\widetilde{u}$ such that the collocation equations

$$
\begin{aligned}
& \mathcal{L} \mathfrak{u}\left(\mathrm{x}_{\mathrm{j}}\right)=\mathcal{L} \widetilde{\mathfrak{u}}\left(\mathrm{x}_{\mathrm{j}}\right)=\mathrm{f}\left(\mathrm{x}_{\mathrm{j}}\right), \quad \mathrm{x}_{\mathrm{j}} \in \mathrm{X}_{1}, \\
& \mathcal{B} u\left(x_{j}\right)=\mathcal{B} \widetilde{u}\left(x_{j}\right)=g\left(x_{j}\right), \quad x_{j} \in X_{2},
\end{aligned}
$$

are satisfied. The resulting linear system that is formed by evaluating (4) and (5) at the collocation points $\mathrm{X}$ with the approximation (7) is of the form $\mathbf{A c}=\mathbf{f}$ where the collocation matrix

$$
\mathbf{A}=\left[\begin{array}{ll}
A_{\mathcal{L L}_{2}} & A_{\mathcal{L B}_{2}} \\
A_{\mathcal{L} \mathcal{L}_{2}} & A_{\mathrm{BB}_{2}}
\end{array}\right]
$$

with entries

$$
\begin{aligned}
& \left(A_{\mathcal{L L}_{2}}\right)_{i j}=\left.\mathcal{L} \mathcal{L}_{2} \Phi(\mathbf{x}, \xi)\right|_{\mathbf{x}=\mathbf{x}_{i}, \xi=\xi_{\mathrm{j}}}, \quad \mathbf{x}_{\mathrm{i}}, \xi_{\mathrm{j}} \in \mathrm{X}_{1}, \\
& \left(A_{\mathcal{L B}_{2}}\right)_{i j}=\left.\mathcal{L} \mathcal{B}_{2} \Phi(\mathbf{x}, \xi)\right|_{\mathbf{x}=\mathbf{x}_{i}, \xi=\xi_{\mathrm{j}}}, \quad \mathbf{x}_{\mathrm{i}} \in X_{1}, \xi_{\mathbf{j}} \in X_{2}, \\
& \left(A_{B \mathcal{L}_{2}}\right)_{i j}=\left.\mathcal{B} \mathcal{L}_{2} \Phi(\mathbf{x}, \xi)\right|_{\mathbf{x}=\mathbf{x}_{i}, \xi=\xi_{j}}, \quad \mathbf{x}_{i} \in X_{2}, \xi_{j} \in X_{1}, \\
& \left(A_{B_{2}}\right)_{i j}=\left.\mathcal{B B}_{2} \Phi(\mathbf{x}, \xi)\right|_{\mathbf{x}=\mathbf{x}_{i}, \xi=\xi_{j}}, \quad \mathbf{x}_{i}, \xi_{j} \in X_{2} .
\end{aligned}
$$

The vector $\mathbf{f}$ consists of the entries $f\left(x_{i}\right), x_{i} \in X_{1}$, followed by entries $g\left(x_{i}\right)$, $\mathbf{x}_{i} \in X_{2}$. We note that $\mathfrak{u}, \widetilde{u}$ are elements of $H^{\tau}(\Omega)$. Under the assumption 
that the functionals $\left\{\lambda_{1}, \ldots, \lambda_{\mathrm{N}}\right\}$,

$$
\begin{aligned}
& \lambda_{j}(u):=\delta_{x_{j}} \circ \mathcal{L}(u)=(\mathcal{L} u)\left(x_{j}\right), \quad j=1, \ldots, n, \\
& \lambda_{j}(u):=\delta_{x_{j}} \circ \mathcal{B}(u)=(\mathcal{B} u)\left(x_{j}\right), \quad j=n+1, \ldots, N,
\end{aligned}
$$

are linearly independent, the symmetric collocation matrix is nonsingular and there exists an unique approximation satisfying the collocation conditions (8) and (9) [12, Section 16.3].

The error between the solution and the approximate solution depends on the mesh norms of the interior and boundary centres, as given in the following lemma.

Lemma 3. Assume that the exact solution of (4) belongs to $\mathrm{H}^{\tau}(\Omega)$ with $\tau>\mathrm{m}+\mathrm{d} / 2$. Let $\mathrm{h}_{1}$ be the mesh norm of the interior collocation points $\mathrm{X}_{1}$, let $\Phi$ be a positive definite kernel satisfying (2) and let $\widetilde{\mathrm{u}}$ be the approximate solution obtained by symmetric collocation. Then we have the error bounds

$$
\|\mathfrak{u}-\widetilde{\mathfrak{u}}\|_{\mathrm{L}_{2}(\Omega)} \leqslant \mathrm{ch}_{1}^{\tau-\mathfrak{m}}\|\mathfrak{u}-\widetilde{\mathfrak{u}}\|_{\mathrm{H}^{\tau}(\Omega)} \leqslant \mathrm{ch}_{1}^{\tau-\mathfrak{m}}\|\mathfrak{u}\|_{\mathrm{H}^{\tau}(\Omega)},
$$

and

$$
\|u-\widetilde{u}\|_{L_{2}(\partial \Omega)} \leqslant C h_{2}^{\tau-1 / 2}\|u-\widetilde{u}\|_{H^{\tau}(\Omega)} .
$$

Proof: From Giesl and Wendland [6],

$$
\|\mathcal{L} \mathfrak{u}-\mathcal{L} \tilde{u}\|_{L_{2}(\Omega)} \leqslant \mathrm{Ch}_{1}^{\tau-m}\|\mathfrak{u}\|_{H^{\tau}(\Omega)} .
$$

Now with (6) and Lemma 3 we reach

$$
\|\mathfrak{u}-\widetilde{\mathfrak{u}}\|_{\mathrm{L}_{2}(\Omega)} \leqslant \mathrm{C}\|\mathcal{L} \mathfrak{u}-\mathcal{L} \widetilde{\mathfrak{u}}\|_{\mathrm{L}_{2}(\Omega)} \leqslant \mathrm{Ch}_{1}^{\tau-\mathfrak{m}}\|\mathfrak{u}\|_{\mathrm{H}^{\tau}(\Omega)},
$$

which proves the first result. The second result is from Giesl and Wendland [6, Theorem 3.10]. 
Algorithm 1: Multiscale symmetric collocation approximation

Data: $n$, the number of levels;

$\left\{X_{1, i}, X_{2, i}\right\}_{i=1}^{\text {n }}$, the interior and boundary collocation points for each level $i$;

$\left\{h_{1, i}, h_{2, i}\right\}_{i=1}^{n}$, mesh norms at each level, satisfying $c \mu \bar{h}_{i} \leqslant \bar{h}_{i+1} \leqslant \mu \bar{h}_{i}$, where $h_{i}=\max \left(h_{1, i}, h_{2, i}\right)$ with fixed $\mu \in(0,1), c \in(0,1]$ and $h_{1}$

sufficiently small;

$\left\{\delta_{i}\right\}_{i=1}^{n}$, the scale parameters to use at each level, satisfying $\delta_{i}=v \bar{h}_{i}^{1-(2 m+d) /(2 \tau)}$, where $v$ is a fixed constant.

\section{1 begin}

$2 \quad$ Set $\widetilde{u}_{0}=0, f_{0}=f, g_{0}=g$

3 for $i=1,2, \ldots, n$ do

With the scaled kernel $\Phi_{\delta_{i}}$, solve the symmetric collocation linear system

$$
\begin{aligned}
& \mathcal{L} s_{i}(\mathbf{x})=f_{i-1}(\mathbf{x}), \quad \text { for all } \mathbf{x} \in X_{1, i} \\
& \mathcal{B} s_{i}(\mathbf{x})=g_{i-1}(\mathbf{x}), \quad \text { for all } \mathbf{x} \in X_{2, i}
\end{aligned}
$$

Update the solution and residual according to

$$
\begin{aligned}
\widetilde{u}_{i} & =\widetilde{u}_{i-1}+s_{i}, \\
f_{i} & =f_{i-1}-\mathcal{L} s_{i}, \\
g_{i} & =g_{i-1}-\mathcal{B} s_{i} .
\end{aligned}
$$

Result: Approximate solution $\widetilde{u}_{n}$ at level $n$. The error at level $n$ is $e_{n}:=u-\widetilde{u}_{n}$. 
Algorithm 1 is the formal statement of our multiscale algorithm for the symmetric collocation solution of (4) and (5).

The following theorem and corollaries are our main results for the convergence of the multiscale symmetric collocation algorithm.

Theorem 4. Let $\Omega \subseteq \mathbb{R}^{\mathrm{d}}$ be a bounded domain with Lipschitz boundary. Let $\Phi$ be a kernel generating $\mathrm{H}^{\tau}\left(\mathbb{R}^{\mathrm{d}}\right)$ and $\Phi_{\mathrm{j}}$ be defined by (1) with scale factor $\delta_{j}$. Then for Algorithm 1 there exists a constant $\alpha_{1}>0$ such that

$$
\left\|\mathrm{E} e_{j}\right\|_{\Phi_{j+1}} \leqslant \alpha_{1}\left\|\mathrm{E} e_{j-1}\right\|_{\Phi_{j}} \text { for } j=1,2, \ldots,
$$

where $\mathrm{Ee}_{\mathrm{j}}$ is the extension operator defined in Lemma 1 applied to the error at level $\mathbf{j}$ defined in Algorithm 1.

Proof: We write

$$
\left\|E e_{j}\right\|_{\Phi_{j+1}}^{2} \leqslant \frac{1}{c_{1}} \int_{\mathbb{R}^{\mathrm{d}}}\left|\widehat{E e_{j}}(\boldsymbol{\omega})\right|^{2}\left(1+\delta_{j+1}^{2}\|\boldsymbol{\omega}\|_{2}^{2}\right)^{\tau} \mathrm{d} \boldsymbol{\omega}=: \frac{1}{c_{1}}\left(I_{1}+I_{2}\right)
$$

with

$$
\begin{aligned}
& I_{1}:=\int_{\|\boldsymbol{\omega}\|_{2} \leqslant \frac{1}{\delta_{j+1}}}\left|{\widehat{E e_{j}}}_{j}(\boldsymbol{\omega})\right|^{2}\left(1+\delta_{j+1}^{2}\|\boldsymbol{\omega}\|_{2}^{2}\right)^{\tau} \mathrm{d} \boldsymbol{\omega}, \\
& \mathrm{I}_{2}:=\int_{\|\boldsymbol{\omega}\|_{2} \geqslant \frac{1}{\delta_{j+1}}}\left|\widehat{\mathrm{Ee}}_{j}(\boldsymbol{\omega})\right|^{2}\left(1+\delta_{j+1}^{2}\|\boldsymbol{\omega}\|_{2}^{2}\right)^{\tau} \mathrm{d} \boldsymbol{\omega} .
\end{aligned}
$$

Now we consider the first integral, where we use $\delta_{j+1}\|\boldsymbol{\omega}\|_{2} \leqslant 1$ and then Lemma 3 and Lemma 2. This is valid since $s_{j} \in V_{j}$ is the approximate solution with symmetric collocation of $\mathcal{L} e_{j-1}=f_{j-1}$. Then

$$
\begin{aligned}
\mathrm{I}_{1} & \leqslant 2^{\tau} \int_{\|\boldsymbol{\omega}\|_{2} \leqslant \frac{1}{\delta_{j+1}}}\left|\widehat{\mathrm{Ee}} \widehat{e}_{j}(\boldsymbol{\omega})\right|^{2} \mathrm{~d} \boldsymbol{\omega} \leqslant 2^{\tau}\left\|\mathrm{E} e_{j}\right\|_{\mathrm{L}_{2}\left(\mathbb{R}^{\mathrm{d}}\right)}^{2} \leqslant \mathrm{C}\left\|e_{j}\right\|_{\mathrm{L}_{2}(\Omega)}^{2} \\
& \leqslant C h_{1, j}^{2 \tau-2 \mathrm{~m}}\left\|e_{j-1}\right\|_{\mathrm{H}^{\tau}(\Omega)}^{2} \leqslant C h_{1, j}^{2 \tau-2 m} \delta_{j}^{-2 \tau}\left\|E e_{j-1}\right\|_{\Phi_{j}}^{2} \\
& \leqslant C v^{-2 \tau}\left\|E e_{j-1}\right\|_{\Phi_{j}}^{2},
\end{aligned}
$$


where we have also used Lemma 3. For the second integral $I_{2}$ we use

$$
\delta_{j+1} / \delta_{j}=\left(\bar{h}_{j+1} / \bar{h}_{j}\right)^{1-(2 m+d) /(2 \tau)} \leqslant \mu^{1-(2 m+d) /(2 \tau)},
$$

and since $\delta_{j+1}\|\boldsymbol{\omega}\|_{2} \geqslant 1$,

$$
\left(1+\delta_{j+1}^{2}\|\boldsymbol{\omega}\|_{2}^{2}\right)^{\tau} \leqslant 2^{\tau} \delta_{j+1}^{2 \tau}\|\boldsymbol{\omega}\|_{2}^{2 \tau} \leqslant 2^{\tau} \mu^{2 \tau-2 m-d}\left(1+\delta_{j}^{2}\|\boldsymbol{\omega}\|_{2}^{2}\right)^{\tau} .
$$

Then using (3) we obtain

$$
I_{2} \leqslant 2^{\tau} c_{2} \mu^{2 \tau-2 m-d}\left\|E e_{j}\right\|_{\Phi_{j}}^{2} \leqslant 2^{\tau} c_{2} \mu^{2 \tau-2 m-d}\left\|E e_{j-1}\right\|_{\Phi_{j}}^{2},
$$

since the interpolant is norm-minimal with respect to the $\mathcal{N}_{\Phi_{j}}\left(\mathbb{R}^{\mathrm{d}}\right)$-norm. Combining our results for $I_{1}$ and $I_{2}$ and now writing $C_{11}$ and $C_{12}$ for the two constants appearing in the bounds of the expressions for $\mathrm{I}_{1}$ and $\mathrm{I}_{2}$, respectively, we find that

$$
\left\|E e_{j}\right\|_{\Phi_{j+1}}^{2} \leqslant\left(v^{-2 \tau} C_{11} / c_{1}+\mu^{2 \tau-2 m-d} C_{12} / c_{1}\right)\left\|E e_{j-1}\right\|_{\Phi_{j}}^{2},
$$

and the result follows with

$$
\alpha_{1}:=\left(v^{-2 \tau} C_{11} / c_{1}+\mu^{2 \tau-2 m-d} C_{12} / c_{1}\right)^{1 / 2} .
$$

Corollary 5. There exist constants $\mathrm{C}_{13}>0$ and $\mathrm{C}_{14}>0$ such that for the solutions of the multiscale symmetric collocation from Algorithm 1 we have the following error bounds

$$
\left\|\mathfrak{u}-\widetilde{\mathrm{u}}_{\mathfrak{n}}\right\|_{\mathrm{L}_{2}(\Omega)} \leqslant \mathrm{C}_{13} \alpha_{1}^{\mathfrak{n}}\|\mathrm{u}\|_{\mathrm{H}^{\tau}(\Omega)} \quad \text { for } \mathrm{n}=1,2, \ldots,
$$

and

$$
\left\|\mathrm{u}-\widetilde{\mathrm{u}}_{\mathfrak{n}}\right\|_{\mathrm{L}_{2}(\partial \Omega)} \leqslant \mathrm{C}_{14} \alpha_{1}^{\mathfrak{n}}\|\mathrm{u}\|_{\mathrm{H}^{\tau}(\Omega)} \quad \text { for } \mathrm{n}=1,2, \ldots .
$$

Thus $\widetilde{\mathbf{u}}_{\mathrm{n}}$ resulting from Algorithm 1 converges linearly to $\boldsymbol{u}$ in the $\mathrm{L}_{2}$-norm in $\Omega$ and on $\partial \Omega$ if $\alpha_{1}<1$. 
Proof: We first consider the solution in $\Omega$. Using Lemma 3 and (3),

$$
\begin{aligned}
\left\|u-\widetilde{u}_{n}\right\|_{L_{2}(\Omega)} & =\left\|e_{\mathfrak{n}}\right\|_{L_{2}(\Omega)} \leqslant C h_{1, n}^{\tau-m}\left\|e_{n-1}\right\|_{H^{\tau}(\Omega)} \\
& \leqslant C \bar{h}_{n}^{\tau-m} \delta_{n+1}^{-\tau}\left\|E e_{n}\right\|_{\Phi_{n+1}} \leqslant C\left\|E e_{n}\right\|_{\Phi_{n+1}} \\
& \leqslant C \alpha_{1}^{\mathfrak{n}}\|u\|_{\Phi_{1}} \leqslant C \alpha_{1}^{\mathfrak{n}}\|u\|_{H^{\tau}(\Omega)}
\end{aligned}
$$

since

$$
\bar{h}_{n}^{\tau-m} \delta_{n+1}^{-\tau}=v^{-\tau} \bar{h}_{n}^{\tau-m} \bar{h}_{n+1}^{-\tau+m+d / 2} \leqslant v^{-\tau}\left(\frac{\bar{h}_{n}}{\bar{h}_{n+1}}\right)^{\tau-m} \leqslant v^{-\tau}(c \mu)^{m-\tau} .
$$

With (11), the proof for the second result follows in an identical fashion to the proof for the first result. In this case we need

$$
\mathrm{h}_{2, \mathrm{n}}^{\tau-1 / 2} \delta_{n+1}^{-\tau} \leqslant v^{-\tau} \bar{h}_{\mathrm{n}}^{\tau-1 / 2} \bar{h}_{n+1}^{-\tau+1 / 2} \leqslant C(c \mu)^{1 / 2-\tau} .
$$

Corollary 6. There exist constants $\mathrm{C}_{15}>0$ and $\mathrm{C}_{16}>0$ such that for the solutions of the multiscale symmetric collocation algorithm we have the following error bounds

$$
\left\|u-\widetilde{u}_{n}\right\|_{L_{\infty}(\Omega)} \leqslant C_{15} \alpha_{1}^{n}\|u\|_{H^{\tau}(\Omega)}, \quad \text { for } n=1,2, \ldots,
$$

and

$$
\left\|\mathrm{u}-\widetilde{\mathrm{u}}_{\mathrm{n}}\right\|_{\mathrm{L}_{\infty}(\partial \Omega)} \leqslant \mathrm{C}_{16} \alpha_{1}^{\mathfrak{n}}\|\mathrm{u}\|_{\mathrm{H}^{\tau}(\Omega)}, \quad \text { for } \mathrm{n}=1,2, \ldots .
$$

Thus $\widetilde{\mathbf{u}}_{\mathrm{n}}$ resulting from Algorithm 1 converges linearly to $\boldsymbol{u}$ in the $\mathrm{L}_{\infty}$-norm in $\Omega$ and on $\partial \Omega$ if $\alpha_{1}<1$.

Proof: The proofs are very similar to the previous corollary and we only highlight the differences in both cases. In $\Omega$,

$$
\left\|u-\widetilde{u}_{n}\right\|_{L_{\infty}(\Omega)} \leqslant C\left\|\mathcal{L} u-\mathcal{L} \widetilde{u}_{n}\right\|_{L_{\infty}(\Omega)},
$$


if we assume that the coefficients of $\mathcal{L}$ are in, say, $\mathrm{C}^{\mathrm{m}}$. Then, from Giesl and Wendland [6],

$$
\|\mathcal{L} u-\mathcal{L} \widetilde{u}\|_{L_{\infty}(\Omega)} \leqslant C h_{1}^{\tau-m-d / 2}\|u\|_{H^{\tau}(\Omega)},
$$

and since

$$
h_{1, n}^{\tau-m-d / 2} \delta_{n+1}^{-\tau}=v^{-\tau}\left(\frac{\bar{h}_{n}}{\bar{h}_{n+1}}\right)^{\tau-m-d / 2} \leqslant C(c \mu)^{\tau-m-d / 2},
$$

the result follows. On $\partial \Omega$ we need to use [6, Theorem 3.10]

$$
\|u-\widetilde{u}\|_{L_{\infty}(\partial \Omega)} \leqslant C h_{2}^{\tau-d / 2}\|u-\widetilde{u}\|_{H^{\tau}(\Omega)},
$$

and

$$
h_{2, n}^{\tau-d / 2} \delta_{n+1}^{-\tau} \leqslant v^{-\tau} \bar{h}_{n}^{\tau-d / 2} \bar{h}_{n+1}^{-\tau+d / 2} \leqslant C(c \mu)^{-\tau+d / 2} .
$$

\section{Numerical experiments}

In this section we present results from applying the symmetric collocation algorithm to the following Poisson problem with Dirichlet boundary conditions [4],

$$
\begin{aligned}
\nabla^{2} u(x, y) & =-\frac{5}{4} \pi^{2} \sin (\pi x) \cos \left(\frac{\pi y}{2}\right), \quad(x, y) \in \Omega:=[0,1]^{2}, \\
u(x, y) & =\sin (\pi x), \quad(x, y) \in \Gamma_{1}:=\{(x, y): 0 \leqslant x \leqslant 1, y=0\}, \\
u(x, y) & =0, \quad(x, y) \in \Gamma_{2}:=\partial \Omega \backslash \Gamma_{1} .
\end{aligned}
$$

The exact solution is

$$
u(x, y)=\sin (\pi x) \cos \left(\frac{\pi y}{2}\right) .
$$


Table 1: The number of equally spaced points used at each level and the associated mesh norm for the numerical experiments.

\begin{tabular}{cccccc} 
Level & 1 & 2 & 3 & 4 & 5 \\
\hline $\mathrm{N}$ & 25 & 81 & 289 & 1089 & 4225 \\
$\overline{\mathrm{h}}$ & $1.75 \times 10^{-1}$ & $8.76 \times 10^{-2}$ & $4.37 \times 10^{-2}$ & $2.19 \times 10^{-2}$ & $1.09 \times 10^{-2}$
\end{tabular}

This same problem, with different scaling parameters $\delta_{i}$ and point sets, was discussed by Fasshauer [4, Table 41.4] where convergence was observed essentially only for a few levels. This was because they used

$$
\delta_{i}=C \bar{h}_{i},
$$

whilst Algorithm 1 uses

$$
\delta_{i}=v \bar{h}_{i}^{1-(2 m+d) /(2 \tau)} .
$$

Fasshauer's [4] choice of $\delta_{i}$ does not result in linear convergence since (16) is no longer valid. This indicates the importance of the theoretical results given in this article.

We used the $\mathrm{C}^{6}$ Wendland RBF

$$
\Phi(\mathbf{x})=(1-\|\mathbf{x}\|)_{+}^{8}\left(32\|\mathbf{x}\|^{3}+25\|\mathbf{x}\|^{2}+8\|\mathbf{x}\|+1\right),
$$

which is positive definite on $\mathbb{R}^{2}[12$, e.g.]. We used five levels for the approximation, with equally spaced point sets at each level. The number of points $\mathrm{N}$ and the mesh norms $h$ are given in Table 1 . The mesh norms decrease by almost exactly one half at each level and hence we select $\mu=\frac{1}{2}$. There are also $4(\sqrt{N}-1)$ equally spaced boundary centres.

The scaling factors used, as specified by Algorithm 1, are given in Table 2 with $v=3.58$. The $\mathrm{L}_{2}$ error was estimated using Gaussian quadrature with a $300 \times 300$ tensor product grid of Gauss-Legendre points and the $\mathrm{L}_{\infty}$ error was estimated with the same tensor product grid. Since the collocation 
Table 2: The scaling factors, approximation errors and condition numbers of the collocation matrices for the multiscale symmetric collocation algorithm example.

\begin{tabular}{cccccc} 
Level & 1 & 2 & 3 & 4 & 5 \\
\hline$\delta_{j}$ & 2 & 1.59 & 1.26 & 1 & 0.79 \\
$\left\|e_{j}\right\|_{2}$ & $3.32 \mathrm{E}-3$ & $1.69 \mathrm{E}-4$ & $1.50 \mathrm{E}-5$ & $8.58 \mathrm{E}-7$ & $3.94 \mathrm{E}-8$ \\
$\left\|e_{j}\right\|_{\infty}$ & $6.06 \mathrm{E}-3$ & $8.52 \mathrm{E}-4$ & $6.20 \mathrm{E}-5$ & $5.80 \mathrm{E}-6$ & $5.38 \mathrm{E}-7$ \\
$\mathrm{~K}_{\mathrm{j}}$ & $1.18 \mathrm{E}+6$ & $2.27 \mathrm{E}+8$ & $4.23 \mathrm{E}+10$ & $6.63 \mathrm{E}+12$ & $1.32 \mathrm{E}+15$
\end{tabular}

matrix (10) is symmetric and positive definite, the conjugate gradient method was used to solve the linear system. In general, some degree of caution is required with the selection of the point sets as the condition number increases with decreasing separation radius [12, Ch. 12].

\section{Conclusion}

We present a theoretical analysis of a multiscale approximation algorithm for constructing numerical solutions to elliptic PDE on a bounded domain. We prove that the approximation converges linearly to the true solution in both the $L_{2^{-}}$and the $L_{\infty}$-norms, in $\Omega$ and on $\partial \Omega$. We explain why a similar algorithm (with different parameter choices), which was studied by Fasshauer [4], did not provide linear convergence. Numerical experiments support the theoretical conclusions.

Acknowledgments We used some datasets and code from Fasshauer [4] as well as Andrea Tagliasacchi's "kd-tree for Matlab" library. ${ }^{1}$

${ }^{1}$ Available at http://www.mathworks.com/matlabcentral/fileexchange/21512. 


\section{References}

[1] S. C. Brenner and L. R. Scott. The Mathematical Theory of Finite Element Methods. Texts in Applied Mathematics. Springer, 3rd edition, 2008. doi:10.1007/978-1-4757-3658-8. C140

[2] C. S. Chen, M. Ganesh, M. A. Golberg, and A. H.-D. Cheng. Multilevel compact radial functions based computational schemes for some elliptic problems. Computers and Mathematics with Applications, 43:359-378, 2002. doi:10.1016/S0898-1221(01)00292-9. C139

[3] A. Chernih and Q. T. Le Gia. Multiscale methods with compactly supported radial basis functions for Galerkin approximation of elliptic PDEs. submitted, 2012. C141

[4] G. E. Fasshauer. Meshfree Approximation Methods with Matlab, volume 6 of Interdisciplinary Mathematical Sciences. World Scientific Publishing Co., Singapore, 2007. C138, C139, C148, C149, C150

[5] G. E. Fasshauer and J. G. Zhang. On choosing 'optimal' shape parameters for RBF approximation. Numerical Algorithms, 45:345-368, 2007. doi:10.1007/s11075-007-9072-8. C138

[6] P. Giesl and H. Wendland. Meshless collocation: Error estimates with application to dynamical systems. SIAM Journal on Numerical Analysis, 45:1723-1741, 2006. doi:10.1137/060658813. C138, C143, C148

[7] Q. T. Le Gia, I. H. Sloan, and H. Wendland. Multiscale RBF collocation for solving PDEs on spheres. Numerische Mathematik, 121:99-125, 2012. doi:10.1007/s00211-011-0428-6. C139

[8] S. Rippa. An algorithm for selecting a good value of the parameter $\mathrm{c}$ in radial basis function interpolation. Advance in Computational Mathematics, 11:193-210, 1999. doi:10.1023/A:1018975909870. C138 
[9] E. M. Stein. Singular Integrals and Differentiability Properties of Functions. Princeton University Press, Princeton, New Jersey, 1970. doi:10.1090/pspum/010/0482394. C140

[10] H. Wendland. Meshless Galerkin methods using radial basis functions. Mathematics of Computation, 68(228):1521-1531, 1998. doi:10.1090/S0025-5718-99-01102-3. C138

[11] H. Wendland. Numerical solution of variational problems by radial basis functions. In Charles K. Chui and Larry L. Schumaker, editors, Approximation Theory IX, Volume 2: Computational Aspects, pages 361-368. Vanderbilt University Press, 1998. C138

[12] H. Wendland. Scattered Data Approximation, volume 17 of Cambridge Monographs on Applied and Computational Mathematics. Cambridge University Press, Cambridge, 2005. doi:10.1017/CBO9780511617539. C138, C140, C143, C149, C150

\section{Author addresses}

1. A. Chernih, School of Mathematics and Statistics, University of New South Wales, Sydney, Australia.

mailto: andrew@andrewch.com

2. Q. T. Le Gia, School of Mathematics and Statistics, University of New South Wales, Sydney, Australia.

mailto:qlegia@unsw.edu . au 